


\title{
Monitoring trabecular bone microdamage using a dynamic acousto-elastic testing method
}

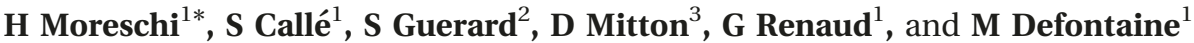 \\ ${ }^{1}$ Université F. Rabelais, Unité 'Imagerie et Cerveau', UMRS/CNRS, Tours, France \\ ${ }^{2}$ Arts et Metiers ParisTech, CNRS/LBM, Paris, France \\ ${ }^{3}$ Université Lyon 1, INRETS/LBMC, Lyon, France
}

The manuscript was received on 17 April 2010 and was accepted after revision for publication on 4 August 2010.

DOI: 10.1243/09544119JEIM846

\begin{abstract}
Dynamic acousto-elastic testing (DAET) is based on the coupling of a lowfrequency (LF) acoustic wave and high-frequency ultrasound (US) pulses (probing wave). It was developed to measure US viscoelastic and dissipative non-linearity in trabecular bone. It is well known that this complex biphasic medium contains microdamage, even when tissues are healthy. The purpose of the present study was to assess the sensitivity of DAET to monitor microdamage in human calcanei. Three protocols were therefore performed to investigate the regional heterogeneity of the calcaneus, the correlation between DAET measurements and microdamage revealed by histology, and DAET sensitivity to mechanically induced fatigue microdamage. The non-linear elastic parameter $\beta$ was computed for all these protocols. The study demonstrated the presence of high viscoelastic and dissipative non-linearity only in the region of the calcaneus close to the anterior talocalcaneal articulation (region of high bone density). Protocols 1 and 2 also showed that most unsorted calcanei did not naturally exhibit high non-linearity, which is correlated with a low level of microcracks. Nevertheless, when microdamage was actually present, high levels of US non-linearity were always found, with characteristic non-linear signatures such as hysteresis and tension/compression asymmetry. Finally, protocol 3 demonstrated the high sensitivity of DAET measurement to fatigue-induced microdamage.
\end{abstract}

Keywords: trabecular bone, microdamage, acousto-elasticity, acoustic non-linearity, viscoelasticity

\section{INTRODUCTION}

Determination of bone strength is a wide and highly complex research domain. It is now clearly understood that bone mineral density (BMD) is not the only factor that may help to predict risk of fracture [1]; among other things, microdamage is suspected of being a significant determinant of bone strength. Microdamage may also have a significant role in the ability of the bone to repair damaged regions $[2,3]$. However, a pathologic imbalance between remodelling and accumulation of microdamage may directly

*Corresponding author: Université F. Rabelais, Unité «Imagerie et Cerveau», équipe 5, UMRS INSERM U930/CNRS ERL 3106, 37032 Tours, France.

email: helene.moreschi@etu.univ-tours.fr impact on the mechanical properties of the bone [4]. In particular, it has not been clearly established whether accumulation of microdamage is a cause or a consequence of the bone remodelling process [5].

Non-invasive clinical characterization of bone strength is currently obtained by dual-energy X-ray absorptiometry (DEXA) measurements and peripheral quantitative computed tomography (pQCT). Both techniques provide essential information on $\mathrm{BMD}$, although $\mathrm{pQCT}$ provides greater space resolution and thus preliminary display of bone microarchitecture. Another approach, namely quantitative ultrasound (QUS), is increasingly being used for the evaluation of bone diseases [6]. Linear QUS techniques have shown their ability to monitor bone changes (microgravity, bed rest) and to detect populations at risk of fracture [7]. However, the ultrasound (US) 
velocity and attenuation (BUA, broadband ultrasound attenuation) measured by these methods are not directly sensitive to the level of bone damage.

The dynamic acousto-elastic testing (DAET) method [8], based on the interaction of two acoustic waves, was recently developed to assess the level of microdamage in trabecular bone samples. In this method, a low-frequency (LF) wave acts as a tension/compression quasi-hydrostatic pressure, and high-frequency US pulses are used as a probe wave to measure the non-linear (NL) viscoelastic and dissipative properties in bone samples.

In the study reported herein, all measurements were made on human calcanei. Although this bone site is not a specific site for osteoporotic fracture, several studies involving QUS measurements have reported that it provides a good indication of fracture risk as well as spine and femur BMD [7, 9, 10].

DAET sensitivity for quantifying bone damage was assessed by three different experimental studies. In view of the heterogeneity of the calcaneus (complex three-dimensional network of trabeculae with several main orientations), DAET measurements were performed in two regions with significant differences in porosity. These two regions were analysed using confocal laser scanning microscopy to correlate the non-linearity measured with the level of microdamage. For the second study, a group of seven parallelepipedic samples were extracted from seven calcanei originating from human donors (principal direction of trabeculae aligned with the physiological loading axis). DAET measurements were performed on each sample before staining for observation by confocal laser scanning microscopy. The third protocol aimed to assess the level of microdamage induced by uniaxial compression fatigue testing. Each sample was measured with the DAET method before and after mechanical testing to follow possible extension of microdamage.

The aim of the present study was thus to assess DAET sensitivity for the measurement of levels of microdamage in trabecular bone tissue, by evaluating the relationship between the classical NL elastic parameter $\beta$ and the level of microdamage.

\section{MATERIALS AND METHODS}

\subsection{Specimen preparation}

Eighteen cancellous bone specimens (from human donors) were divided into three groups, corresponding to the three protocols. Eight whole calcanei were studied in the first protocol. Ten others were cut into parallelepipedic samples: seven were used in the second protocol and three were used in compression fatigue testing.

All calcanei were cleaned of soft tissue and marrow, and defatted using the Supercrit ${ }^{\circledR}$ technique (BioBank, Paris, France) which eliminates fats while protecting the organo-mineral structure of bone tissue (supercritical $\mathrm{CO}_{2}$ delipidation) and its mechanical properties [11]. This cleaning process enables specimens to be kept at room temperature. The Supercrit method of removing the fat from calcanei requires cutting of both lateral cortical faces into planes parallel to the sagittal plane.

One further calcaneus used in the first protocol was depleted of marrow by immersion in hot water and then in trichloroethylene. However, the latter cleaning process causes damage to the collagen of the bone tissue.

\subsection{Dynamic acousto-elastic testing}

An experimental technique, similar to the method proposed by Ichida et al. [12] and named DAET $[\mathbf{8}, \mathbf{1 3}, \mathbf{1 4}]$, was developed to measure viscoelastic and dissipative acoustic non-linearity both noninvasively and regionally.

The DAET experimental configuration (Fig. 1) was based on the interaction of two acoustic waves propagating in perpendicular directions: an LF sinusoidal acoustic wave (long burst of approximately 100 periods) and US pulses with a central frequency of $0.1-1 \mathrm{MHz}$. The trabecular bone sample was placed in the interaction region of the two acoustic beams. The LF acoustic wave selected was between 1 and $5 \mathrm{kHz}$ (typically $2.8 \mathrm{kHz}$ ) so that the corresponding wavelength in water was approximately ten times greater than the US path, according to the assumption of a quasi-static pressure. Furthermore, the US pulses were emitted with a repetition rate approximately ten times higher than the LF wave frequency (typically 20 to $40 \mathrm{kHz}$ ). The US pulses therefore probed the medium at different states of the quasi-hydrostatic pressure: successive tension (expansion) and compression phases. Two long time-domain signals $(80-100 \mathrm{~ms})$ were recorded, i.e. the LF pressure (using an LF hydrophone) and the US pulses. Time-of-flight modulation (TOFM) and relative amplitude modulation (RAM) of US pulses induced by the LF acoustic wave were calculated. TOFM and RAM are associated with viscoelastic and dissipative non-linearity, respectively [13]. 


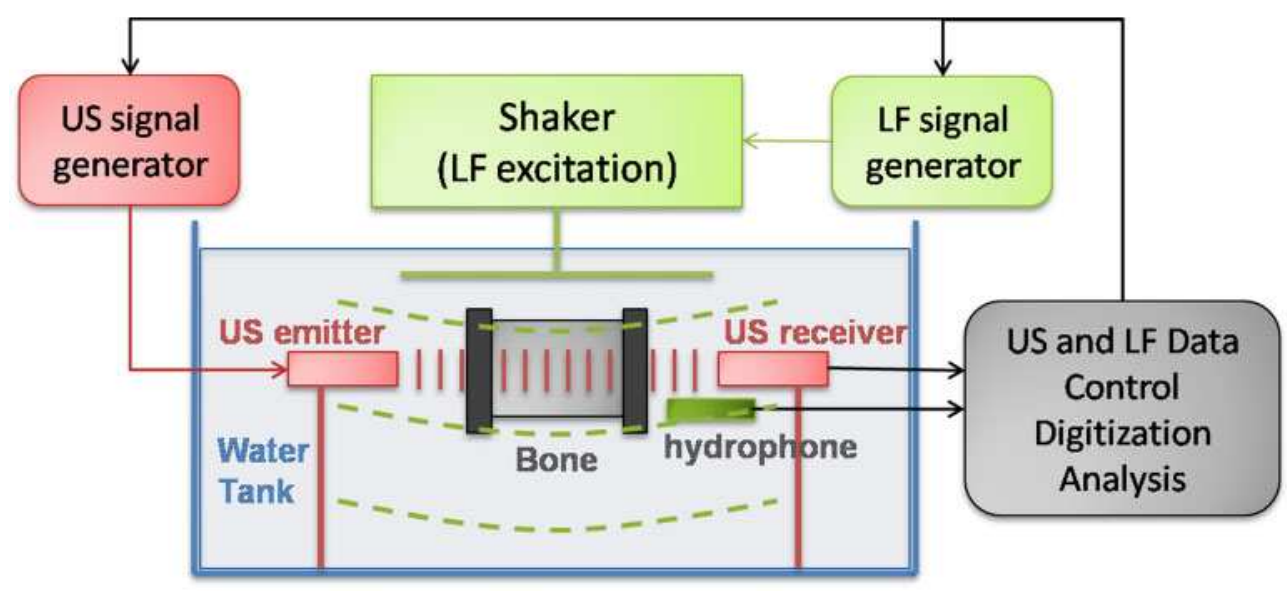

Fig. 1 DAET experimental configuration

The different steps of the data analysis process are illustrated in Fig. 2. Each US pulse-echo was compared in phase $(\triangle \mathrm{TOF})$ and in amplitude with the first referenced pulse (no LF stressed) as presented in Fig. 2(a), leading to the construction of the TOFM and RAM time-domain functions. As expected, in water, TOFM and LF pressure have opposite phases (Fig. 2(b)).

\subsubsection{Viscoelastic non-linearity}

TOFM was obtained from the time position of the maximum of the cross-correlation between the first US pulse signal propagated through the steady medium (no LF stress) and the following pulses (with LF stress). It can be easily established that TOFM (nanoseconds) is proportional to the variations in the viscoelastic modulus $K[\mathbf{1 3}, \mathbf{1 4}]$

$$
\begin{aligned}
\mathrm{TOFM}_{\text {sample }} & =\mathrm{TOF}(i)-\mathrm{TOF}(\mathrm{ref}) \\
& =-\frac{L_{\text {sample }}}{2 \rho_{\text {sample }} c_{\text {sample }}^{3}} \Delta K_{\text {sample }}
\end{aligned}
$$

where $L_{\text {sample }}$ is the US propagation distance in the sample length, $\rho_{\text {sample }}$ and $c_{\text {sample }}$ are the medium density and US velocity in standard conditions (ambient pressure, $20^{\circ} \mathrm{C}$ ), and $\Delta K$ represents the small variations in the elastic modulus.

In a first approximation, the second-order development of Hooke's law was considered

$$
\sigma=K \varepsilon=K_{0}(1+\beta \varepsilon) \varepsilon
$$

This allowed defining the quadratic NL elastic parameter $\beta$, where $\sigma$ and $\varepsilon$ are the stress and strain, respectively. The parameter $\beta$ provides a first quantification of the elastic non-linearity.

\subsubsection{Dissipative non-linearity}

RAM (\%) is the normalized difference in amplitude (Ampli) between the current US pulse and the first reference pulse

$$
\mathrm{RAM}=\frac{\operatorname{Ampli}(i)-\operatorname{Ampli}(\mathrm{ref})}{\operatorname{Ampli}(\mathrm{ref})} \times 100
$$

Instantaneous TOFM and RAM were plotted as a function of the instantaneous LF acoustic pressure (dynamic acousto-elastic diagrams). Figure 3 illustrates such diagrams obtained in water: TOFM was close to $-2 \mathrm{~ns}$ at $50 \mathrm{kPa}$; there was no RAM (noise level).

The quadratic NL elastic parameter $\beta$ was calculated from the linear regression of TOFM in relation to the LF pressure curve as

$$
\beta=-\frac{2 \rho_{\text {sample }} c_{\text {sample }}^{3}}{L_{\text {sample }}} \frac{\Delta \mathrm{TOFM}}{\Delta p_{\mathrm{LF}}}
$$

A positive value of $\beta$ corresponds to a negative slope of the TOFM curve (equation (4)). $\beta=5.6 \pm 0.1$ was measured for water at $21^{\circ} \mathrm{C}$ (straight line in TOFM plot in Fig. 3(a)), close to the value reported in the literature $(\beta=5)$.

In the case of a DAET measurement performed in a bone sample, any non-linearity due to water was subtracted from the measurement of TOFM. However, trabecular bone samples are complex biphasic (water and solid bone tissue) media, whose porosity is not easily defined. In consequence, the calculated value of $\beta$ corresponds to the $\beta$ value of an overall or effective medium (waterlogged sample).

The linear parameters BUA and $c_{\text {sample }}$ were also calculated for each measurement. These two parameters are partly related to the density of the medium: 


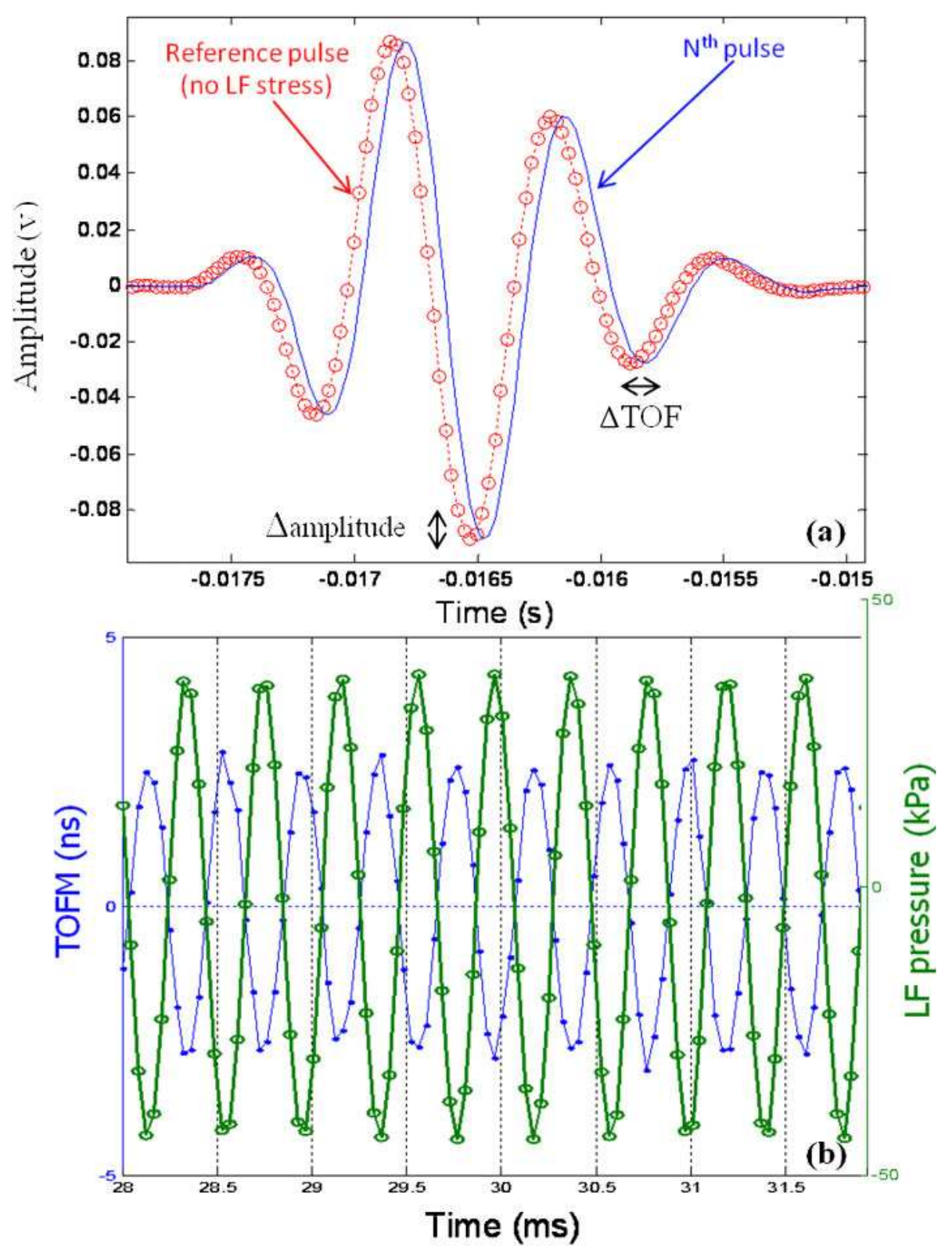

Fig. 2 (a) First (- - $\bigcirc--)$ and Nth (-) US pulse-echoes; (b) TOFM ( $-\bullet$ ) and LF pressure $(-\bigcirc-)$ time-domain functions obtained in water

the higher the medium density, the higher the BUA and $c_{\text {sample values. }}$

\subsection{Mechanically induced damage}

In the third protocol (section 3.3), specimens were subjected to compression fatigue testing to simulate microcracks created during physiological cyclic loading [15]. Three samples of calcanei from three human donors were machined into parallelepipeds. In the same way as before, the main axis of the specimen was approximately aligned with the physiological loading of the calcaneus bone. Sample ends were embedded in Araldite ${ }^{\circledR}$ epoxy resin (end caps) to minimize the effects of end artefacts on mechanical testing.

Compression fatigue tests were carried out in an INSTRON Electropuls E1000 testing machine at ambient temperature. The three specimens, moisturized the day before, were preloaded at $30 \mathrm{~N}$ to ensure full contact. The frequency of loading was fixed at $2 \mathrm{~Hz}$ (near to gait frequency). Pre-cycling was performed between strains of 0.2 and 0.6 per cent for 60 cycles to measure the initial Young's modulus. Young's modulus was evaluated using the most linear part of the loading curve. Load-controlled fatigue was then applied with a load normalized 


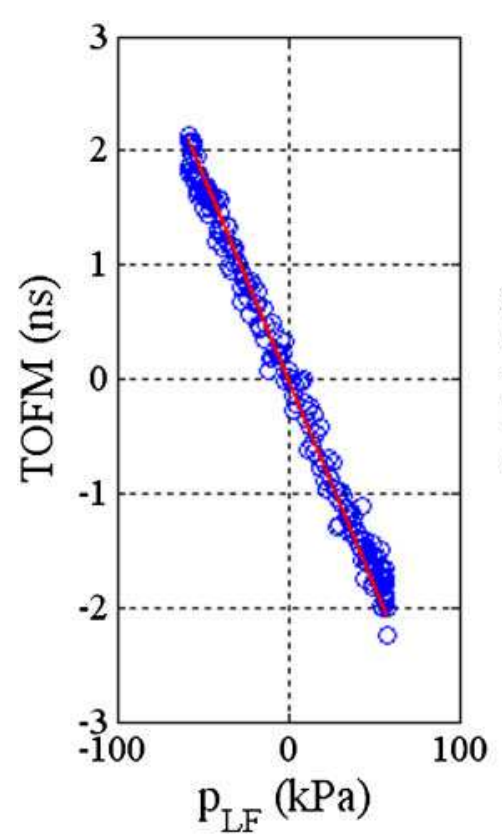

(a)

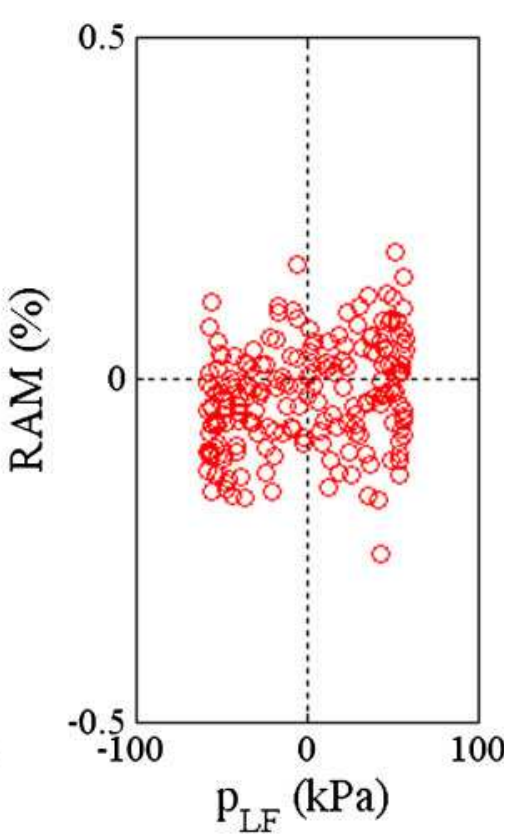

(b)

Fig. 3 (a) TOFM and (b) RAM in water compared to instantaneous LF pressure. The straight line in Fig. 3(a) was plotted using the $\beta$ value reported in the literature (close to 5). There was no RAM in water (noise level)

between 0.2 and 0.6 percent of strain; Young's modulus was calculated throughout the test every 20 cycles and fatigue tests were stopped at 50 per cent reduction in Young's modulus.

\subsection{Histological quantification of damage}

For the first two protocols, a histology analysis was performed by confocal laser scanning microscopy [15], in order to compare US non-linearity levels and microdamage levels. Samples (in sections 3.1 and 3.2) were stained with either alizarin complexone $(0.02$ per cent) or green calcein (0.005 per cent) for $8 \mathrm{~h}$ after DAET measurements. They were then rinsed in deionized water to remove any unbound dye.

These specimens were embedded in Araldite epoxy resin. Two or three 'thin' sections (200$300 \mu \mathrm{m}$ ) of each sample were cut along the US probe axis and mounted on glass slides. Microdamage was studied using a $10 \times$ objective lens and quantified for each slice in a plane parallel to the US pulse propagation direction. A $1 \mathrm{~mm}$ wide region at each side of the section was not analysed to avoid damage induced by sample preparation. Binarization processing of the images from microscopy was used to estimate the relative bone area analysed. Microdamage was quantified for each specimen by measuring crack density, i.e. the number of cracks per relative bone area of the slides studied.

\section{RESULTS}

\subsection{Regional DAET measurements in whole calcanei: influence of trabecular porosity}

\subsubsection{DAET and linear US characterization}

The heterogeneous density distribution and the various trabecular orientations within a calcaneus led to the performance of DAET measurements on whole bones in order to detect levels of non-linearity particular to a given region. Eight trabecular bones were removed from four human donors (all female, 79 to 88 years of age). As shown in Fig. 4, two regions of interest (ROI) were investigated in particular:

(a) a low-porosity region with a mainly plate-like architecture, located in the upper part of the calcaneus;

(b) a higher-porosity homogeneous region, located in the posterior part of the calcaneus (as defined in reference [16]).

Linear BUA and $c_{\text {sample }}$ US parameters were measured and found to be well correlated $\left(r^{2}=0.86\right)$ (Table 1). There was no acoustic non-linearity in region 2. The mean BUA values for all samples were $95 \pm 43 \mathrm{~dB} / \mathrm{MHz}$ in region 1 and $83 \pm 29 \mathrm{~dB} / \mathrm{MHz}$ in region 2 . The velocity was close to $1751 \pm 198 \mathrm{~m} / \mathrm{s}$ in region 1 and $1626 \pm 65 \mathrm{~m} / \mathrm{s}$ in regions 2 . These results clearly indicate the higher inter-specimen 


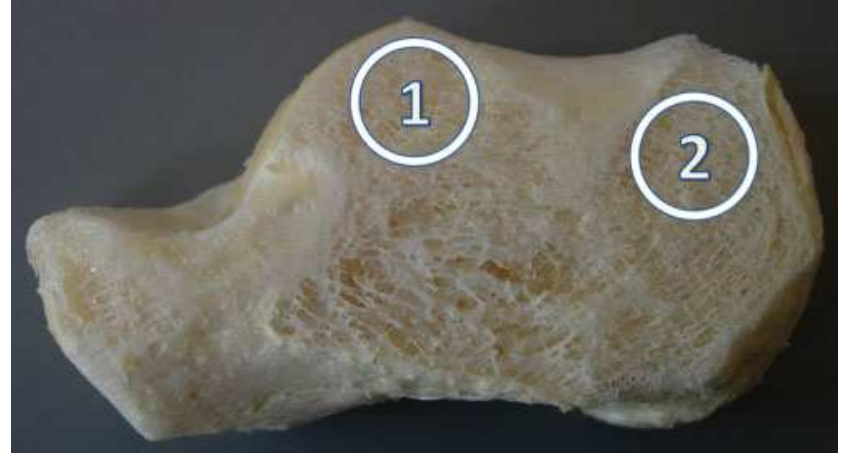

Fig. 4 Two regions measured on human calcanei with DAET. Regions 1 and 2 correspond to the upper (low porosity) and posterior (high porosity) ROI, respectively

variability in region 1 compared with region 2 . Instantaneous TOFM and RAM were plotted and analysed as a function of instantaneous LF pressure (Fig. 5).

The instantaneous TOFM diagram in water was a linear function of LF pressure (Fig. 3(a)) without any hysteresis or strong asymmetry (tension/compression), as expected in a classical NL material. In this case no dissipative non-linearity was observed (noise level). In the presence of microdamage, higher levels of non-linearity were measured, mostly associated with asymmetry and hysteretic behaviour (nonclassical NL behaviour). Moreover, dissipative nonlinearity was always observed. In this case, it was necessary to calculate values of $\beta$ separately in tension $\left(\beta_{\mathrm{t}}\right)$ and compression $\left(\beta_{\mathrm{c}}\right)$ phases (Table 1$)$.

Weak viscoelastic non-linearity was measured in region 2: $\beta=5 \pm 2$ (including calcaneus 7).

Sample 7 in region 1 exhibited very high viscoelastic and dissipative non-linearity (Fig. 5(a)), with a $\beta$ value of $-176 \pm 37$ (average over three measurements). The mean $\beta$ value for the other seven calcanei was $8 \pm 4$.

Except in calcaneus 7, the RAM measured was close to the noise level $( \pm 0.2$ per cent) in both regions 1 and 2. DAET induced opening and closing of the microcracks (LF pressure), leading to attenuation of
US pulses and velocity modulations. RAM was less attenuated in compression than in tension, probably because microcracks more easily open than close.

Since calcaneus 7 exhibited high levels of nonlinearity in region 1 , it was examined by microscopy (in both regions 1 and 2).

In addition to this protocol, DAET measurements were performed on a calcaneus defatted by immersion in a bath of trichloroethylene for a few days (more destructive process). Measurements were performed in region 1 (upper part of the calcaneus). Compared with Fig. 5, a positive $\beta$ value can be seen in Fig. 6, and similar levels of viscoelastic and dissipative non-linearity. Moreover, asymmetry in the TOFM curve can be seen, which means that the sample had two distinct values of the NL $\beta$ parameter: one in tension $\left(\beta_{\mathrm{t}}=162\right)$ and the other in compression $\left(\beta_{\mathrm{c}}=15\right)$.

Confocal laser scanning microscopy was also performed on this sample to evaluate the level of microdamage in the region measured.

\subsubsection{Histology}

Calcaneus 7 and the calcaneus defatted by trichloroethylene were analysed using a laser scanning confocal microscope to compare the DAET results with existing microdamage. Calcaneus 7 was divided into two blocks corresponding to regions 1 and 2, whereas the other calcaneus was analysed by microscopy only in region 1 .

Two types of damage were detected in calcaneus 7:

(a) microdamage in the form of linear microcracks (Figs 7(a) and (b));

(b) macrodamage in the form of split trabeculae, which should not be confused with microfracture (Fig. 7(c)).

To the authors' knowledge, this latter kind of damage has not previously been reported in papers dealing with damage within bone. However, it could be identified with linear cracks described by Moore and Gibson [17].

Table 1 Linear and NL parameters of the specimens measured in region 1

\begin{tabular}{llrrrr}
\hline Sample & $c_{\text {sample }}(\mathrm{m} / \mathrm{s})$ & BUA $(\mathrm{dB} / \mathrm{MHz})$ & $\beta$ & $\beta_{\mathrm{t}}$ & \multicolumn{1}{c}{$\beta_{\mathrm{c}}$} \\
\hline 1 & 1587 & 52 & 6 & 5 & 5 \\
2 & 1590 & 66 & 8 & 8 & 7 \\
3 & 1610 & 70 & 3 & 2 & 4 \\
4 & 2084 & 149 & 14 & 20 & 21 \\
5 & 1566 & 50 & 6 & 6 & 6 \\
6 & 1901 & 128 & -176 & -163 & 8 \\
7 & 1905 & 159 & 12 & 11 & -195 \\
8 & 2101 & 141 & & & 11 \\
\hline
\end{tabular}

$\beta_{\mathrm{t}}, \beta$ calculated in tension phase; $\beta_{\mathrm{c}}, \beta$ calculated in compression phase. 


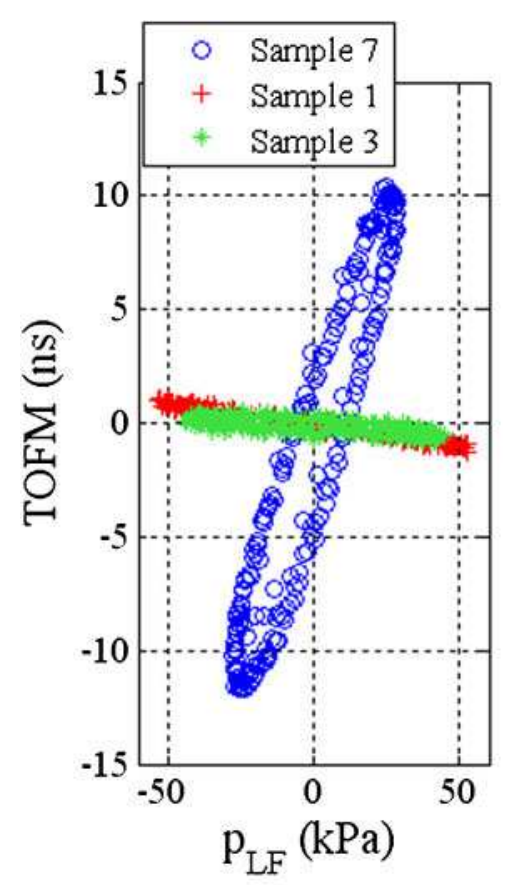

(a)

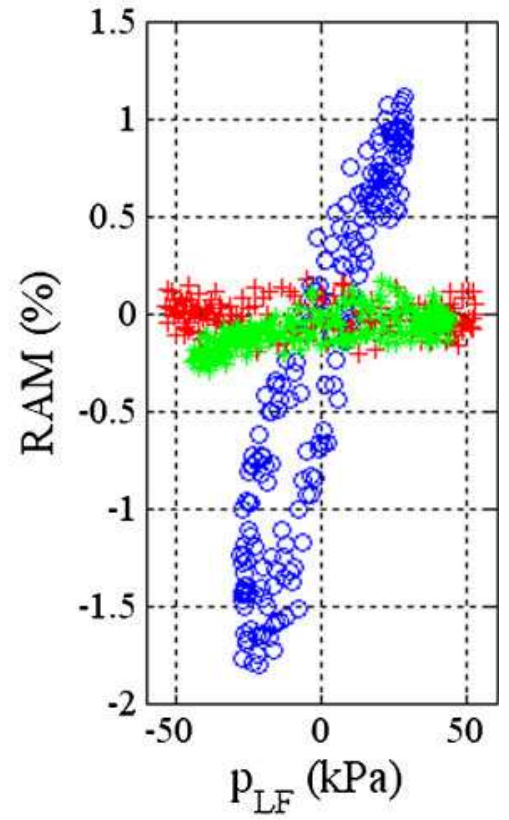

(b)

Fig. 5 Three TOFM (a) and RAM (b) curves measured in region 1 for calcanei 1, 3, and 7. Calcaneus 7 exhibits slight asymmetry between tension and compression phases

DAET may also be sensitive to such splits, and both types of damage were therefore counted separately.

As presented in Table 2, the amount of damage quantified in region 1 was twice as high as in region 2 , in agreement with DAET measurements: viscoelastic and dissipative non-linearity was higher in region 1. Moreover, the difference in bone density (41.6 and 29.0 per cent in regions 1 and 2, respectively) was not

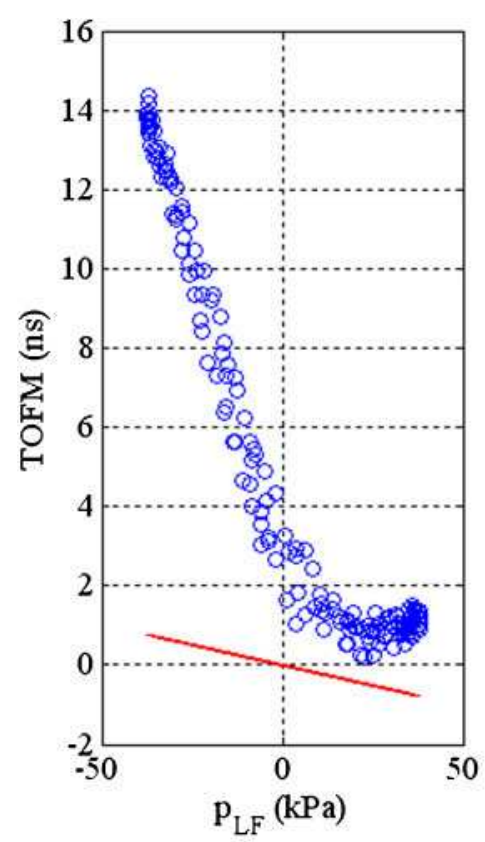

(a)

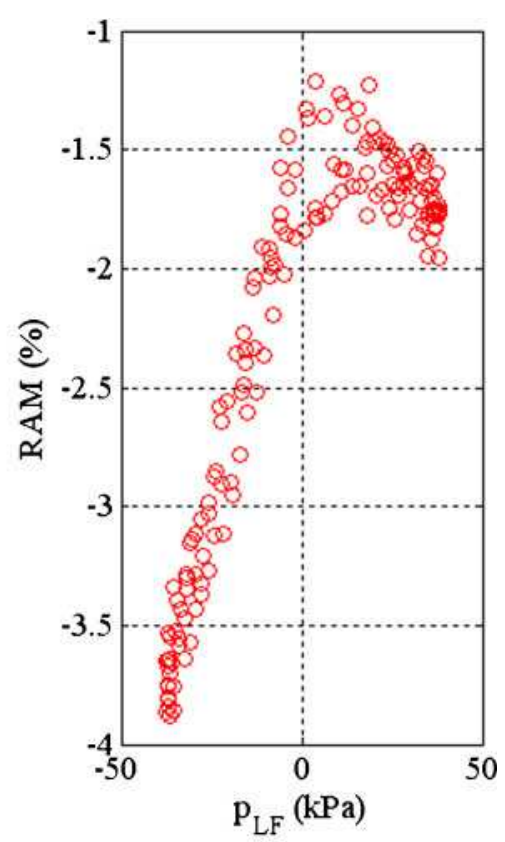

(b)

Fig. 6 (a) TOFM and (b) RAM in relation to LF pressure measured in a calcaneus defatted by trichloroethylene. Asymmetry in tension and compression characterizes NL behaviour that is different in tension and in compression 


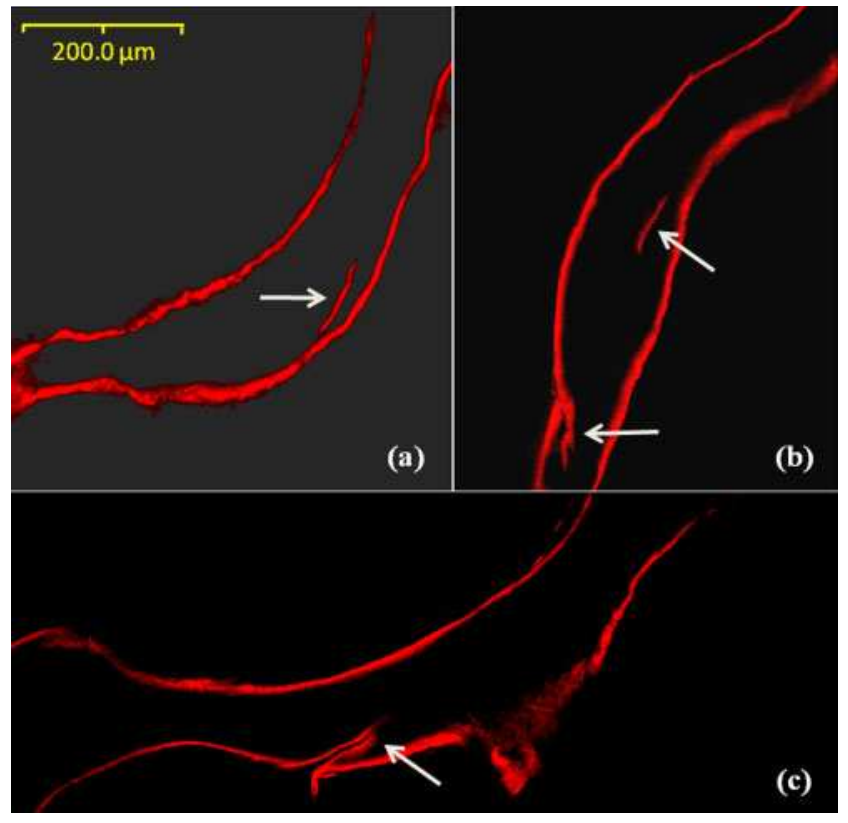

Fig. 7 Examples of damage observed in region 1 by confocal laser scanning microscopy: (a) and (b) two single linear microcracks; (c) split trabeculae

the only explanation for the difference in crack density. Being close to the talus, more stress is concentrated in region 1 during physiological loading than in region 2, which may explain this difference.

The microdamage level observed in confocal images in the trichloroethylene-defatted calcaneus was much higher than had been seen before. There were at least ten times more cracks than in region 1 of sample 7. Several microscopy images of this sample are presented in Fig. 8.

\subsection{DAET in parallelepipedic calcaneal samples extracted along the principal trabecular orientation}

The aim of the second protocol was to establish a relationship between measurements of US non-

Table 2 Mean damage quantification in calcaneus 7 using confocal laser scanning microscopy of three slices for each region

\begin{tabular}{lcc}
\hline & Region 1 & Region 2 \\
\hline CrDn $\left(/ \mathrm{mm}^{2}\right)$ & 0.201 & 0.105 \\
SD & 0.015 & 0.013 \\
SpTDn $\left(/ \mathrm{mm}^{2}\right)$ & 0.263 & 0.109 \\
SD & 0.047 & 0.039 \\
RBAr $(\%)$ & 41.6 & 29.0 \\
\hline
\end{tabular}

CrDn, crack density; SD, standard deviation; SpTDn, surface density of split trabeculae; RBAr, relative bone area. linearity and quantification of microdamage (histology). Seven samples of trabecular bone were taken from seven calcanei from human donors and machined into parallelepipedic specimens. The main sample direction was approximately aligned with the physiological loading axis, which was perpendicular to the direction of observation reported in section 3.1. In order to obtain an acceptable signal-to-noise ratio (SNR) for the US pulses received $(\geqslant 30 \mathrm{~dB}$ ), specimen length ranged from 20 to $25 \mathrm{~mm}$.

\subsubsection{DAET}

At least three DAET lengthwise measurements were performed in bone samples, with sample repositioning between each measurement. Only one sample (sample Z) differed from the others, providing very high TOFM levels (Fig. 9). The other six samples did not display any asymmetry in the TOFM curve, and only mean values of $\beta$ are listed in Table 3 .

\subsubsection{Histology}

To relate the acoustic non-linearity and the actual microdamage objectively, samples were stained according to the labelling protocol described in section 2 and embedded in Araldite epoxy resin. Two thin sections were cut from each sample along the US probe axis. Microdamage was observed under confocal epifluorescence microscopy as described in section 2. Only sample $\mathrm{Z}$ exhibited a high level of microdamage $\left(1.175 \pm 0.090\right.$ per $\mathrm{mm}^{2}$, all damage taken together, whereas quantified damage in the other samples did not exceed $0.563 \pm 0.010$ per $\mathrm{mm}^{2}$, see Table 3). The same specimen $\mathrm{Z}$ also differed from the others in DAET measurements (hysteresis).

After sample $\mathrm{Z}$, two samples ( $\mathrm{M}$ and $\mathrm{X}$ ) provided the highest values of parameter $\beta$, which were quite low values in relation to crack density. However, the microcracks observed were very different from the damage previously described and identified. Most of the quantified damage was due to stained cement lines which were clearly different from microcracks.

\subsection{Sensitivity of DAET to mechanically induced damage}

The goal of this pre-study was to investigate the sensitivity of the DAET technique to microdamage induced by uniaxial compression fatigue testing. 


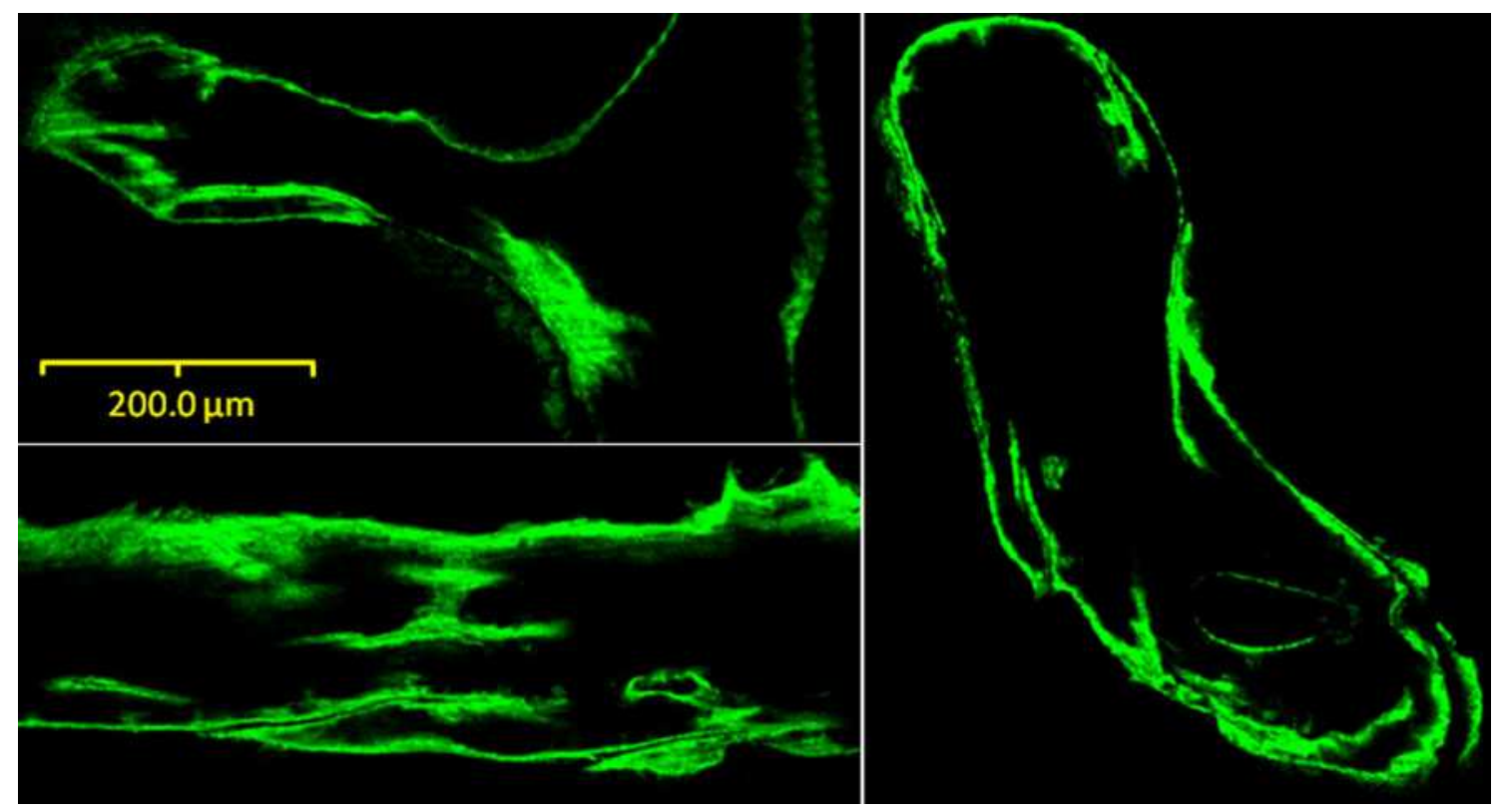

Fig. 8 Examples of images of damage in the calcaneus defatted by trichloroethylene performed by confocal laser scanning microscopy

\subsubsection{Fatigue testing}

Three specimens (A, B, and C) were damaged mechanically, and their results are presented in Table 4 . As reported in Fig. 10, the mechanical experiments induced damage that could be assessed by the ratio of the initial Young's modulus to the current Young's modulus.
Table 4 summarizes the different fatigue test characteristics, including initial Young's modulus, fatigue loading range, and number of cycles to failure. The measured value of the $\beta$ parameter is also included. The observed high difference in the number of loading cycles between samples, particularly for sample A, can be first explained by the high inter-specimen variability. In addition, the samples

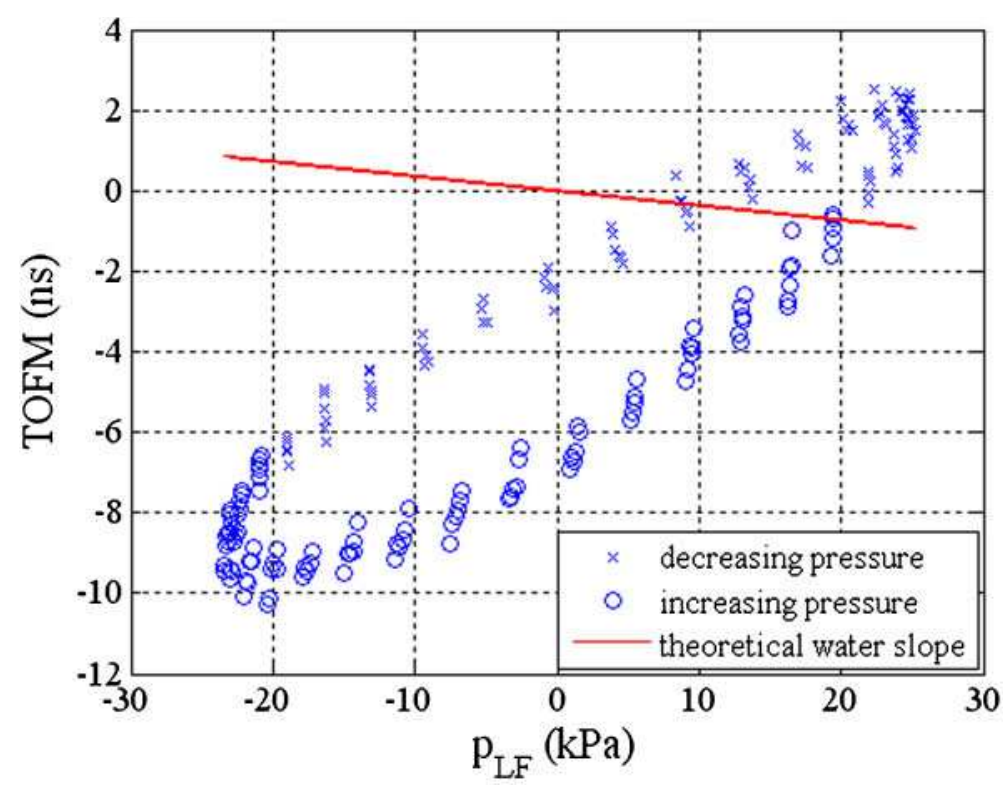

Fig. 9 TOFM in relation to LF pressure for the one parallelepipedic calcaneal sample that exhibited strong non-linear behaviour. The positive slope reveals a negative value of $\beta$ 
Table 3 Quantification of damage averaged over two slices and the mean $\beta$ value over three DAET measurements for seven samples of calcaneal trabecular bone

\begin{tabular}{lllr}
\hline Sample & CrDn $\left(/ \mathrm{mm}^{2}\right)$ & $\mathrm{SD}$ & $\langle\beta>$ \\
\hline Q & 0.123 & 0.030 & 4 \\
R & 0.080 & 0.060 & 6 \\
T & 0.155 & 0.080 & 4 \\
P & 0.115 & 0.080 & 0 \\
M & 0.381 & 0.120 & 31 \\
X & 0.563 & 0.010 & 14 \\
Z & 1.175 & 0.090 & -152 \\
\hline
\end{tabular}

CrDn, crack density; SD, standard deviation; $\langle\beta>$, mean of $\beta$.

size is also big enough to include heterogeneous porosity regions.

\subsubsection{DAET}

DAET measurements were performed three times on samples before and after mechanical testing. All specimens exhibited strong NL behaviours that were very different from those of water. TOFM amplitudes for sample A were slightly higher after compression fatigue testing and RAM was identical before and after fatigue testing, whereas TOFM amplitudes for samples B and C were much higher after mechanical testing (Fig. 11). Sample B also exhibited significant hysteresis after fatigue testing, possibly characterizing significant non-classical hysteretic non-linearity such as microcracks.

\section{DISCUSSION}

The current study focused on the ability of the DAET method to detect microdamage in the human calcaneus. US viscoelastic and dissipative nonlinearity was therefore measured in several trabecular bone samples in three different protocols.

\subsection{Little intrinsic acoustic non-linearity in calcaneus}

The heterogeneity of the calcaneus was first investigated with regard to NL US measurements (protocol 1). In particular, the impact of trabecular bone porosity on DAET measurements was assessed in two different porosity regions. Two calcanei out of nine exhibited very high US non-linearity in the lowporosity region, being one order of magnitude higher than the other calcanei for TOFM (15 to $20 \mathrm{ns,}$

Table 4 Mechanical fatigue testing parameters and DAET $\beta$ measurements for three calcaneal samples

\begin{tabular}{lllrrr}
\hline Sample & $E_{0}(\mathrm{MPa})$ & Load range $(\mathrm{N})$ & $N_{\mathrm{f}}$ & $\beta_{\text {before }}$ & $\beta_{\text {after }}$ \\
\hline A & 274 & $52-257$ & 24470 & -1 & -31 \\
B & 268 & $61-283$ & 504 & -132 & -1066 \\
C & 510 & $44-475$ & 713 & 26 & -524 \\
\hline
\end{tabular}

$E_{0}$, initial Young's modulus; $N_{\mathrm{f}}$, number of cycles to failure; $\beta_{\text {before, }} \beta$ value measured before mechanical fatigue testing; $\beta_{\text {after, }} \beta$ value measured after mechanical fatigue testing.

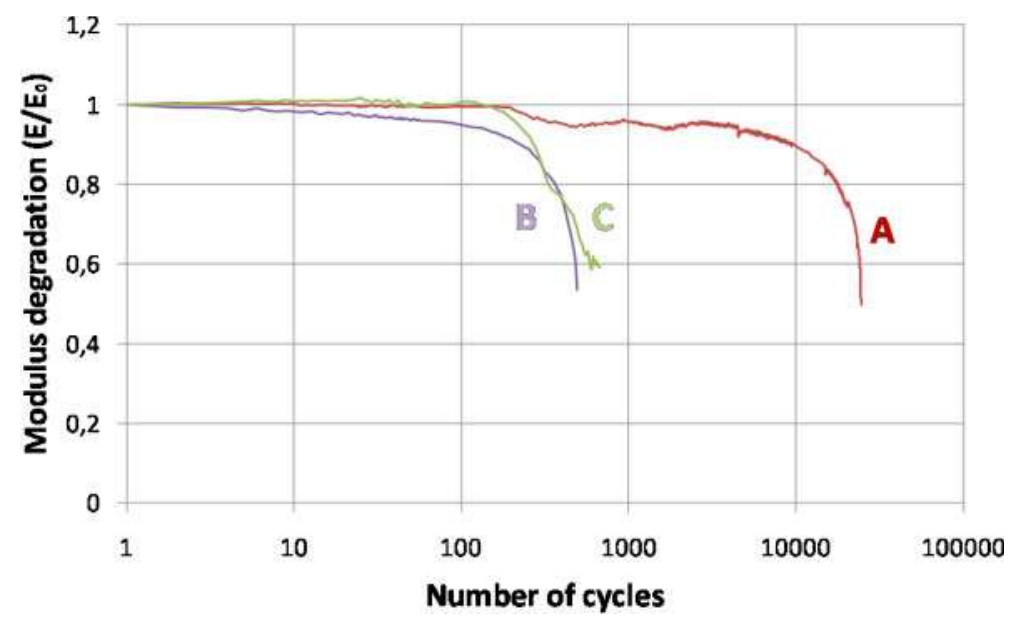

Fig. 10 Evolution of Young's modulus for the three samples 
(A)
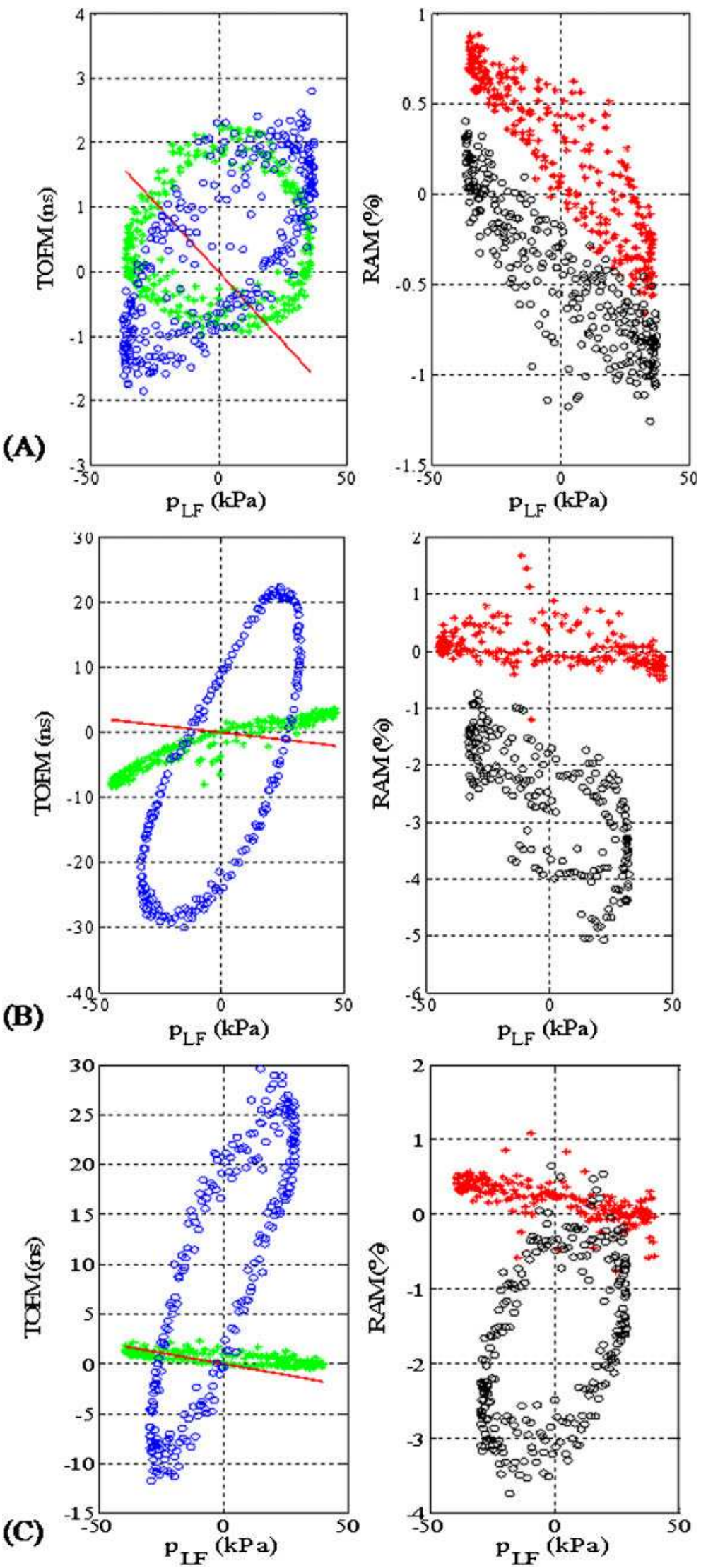

Fig. 11 TOFM and RAM compared to LF pressure before $(*)$ and after $(\bigcirc)$ uniaxial compression fatigue testing for the three samples (A, B, and C). Straight line = theoretical TOFM in water 
$|\beta| \gg 50)$ and more than 2 per cent for RAM. In both cases, the corresponding bone measurement region was close to the anterior talocalcaneal articulation. This is a dense region of the calcaneus, accumulating significant physiological stress and thus a possibly higher level of microdamage. The other seven calcanei tested exhibited low levels of acoustic non-linearity in this upper dense region, explained either by a low level of microdamage and/or by the sensitivity of the DAET to detect low levels of microdamage. The sensitivity of the DAET method relies on a very good US pulse SNR. The accuracy of the cross-correlation technique used to assess the TOFM is in fact highly dependent on the SNR value. High bone density regions obviously lead to poorer SNR. If the level of microdamage is not high enough the TOFM is close to the noise level.

In the higher-porosity region in the lower posterior part of the calcaneus the DAET measurements exhibited very low non-linearity for TOFM ( 1 to $2 \mathrm{~ns}$, $|\beta| \ll 10$ ) and for RAM (lower than 0.5 per cent). This lower US non-linearity, close to that of water, may be explained by either a smaller amount of bone tissue probed by the US pulses and/or to a lower density of microcracks.

Confocal laser scanning microscopy histology performed on calcaneus 7 defatted using the Supercrit method (low- and high-porosity regions) and on the trichloroethylene-defatted calcaneus supports these observations. In particular, it was shown that a high level of microdamage is well correlated with high and complex (asymmetry, hysteresis) NL responses.

In addition to these results, different NL behaviours were observed in the low-porosity region of calcaneus 7 and in the trichloroethylene-defatted calcaneus. The former exhibited high negative $\beta$ values and the latter positive values. This was probably due to the defatting process, which in the first case ensured minimal denaturation of the organo-mineral structure compared with the latter. Clarification of this is being addressed in a further study.

\subsection{Acoustic non-linearity correlated well with microdamage in calcaneal trabecular bone}

The second protocol was performed to confirm the relationship between DAET measurements and damage using confocal laser scanning microscopy analysis on eight parallelepipedic trabecular bone samples (22 mm length) extracted from Supercritdefatted calcanei. This study showed a good correlation between high levels of viscoelastic and dissipative non-linearity and high levels of damage.
Although only one sample out of eight exhibited high and complex NL behaviour, the crack density of this sample was twice as high as the others. It should be noted that sections were cut according to the main physiological loading axis. The density of the trabecular network was therefore fairly heterogeneous and differed slightly from one specimen to another. Here again, amplitudes of acoustic nonlinearity are extremely dependent on factors such as the SNR of US pulses, the amount of bone material probed by the US pulses, and the level of damage.

Solid non-damaged materials exhibit classical NL $\beta$ values of up to 15 . When such media include microdamage, $\beta$ values are very high and mostly associated with non-classical hysteretic NL behaviours in rocks [18-20] and cortical bone tissue [21, 22]; as for example in good agreement with our results in protocols 1 and 2. In all the samples probed with the DAET method, very few (three to five out of 17) exhibited high levels of non-linearity. The native microdamage in trabecular bone does not seem that high. The question to be answered in the study was to identify the threshold of microdamage to which DAET is sensitive and whether it corresponds to a risk of fracture. To attempt to provide answers to this question a third DAET protocol was performed on three trabecular bone samples before and after a mechanical fatigue test aiming to induce damage.

\subsection{DAET sensitivity to mechanically induced microdamage}

Despite the variations between the specimens in terms of number of cycles to failure (even for similar initial Young's moduli) and the limited number of specimens, the results from this preliminary fatigue protocol suggest that DAET is sensitive to mechanically induced damage. Viscoelastic and dissipative non-linearity increased with accumulation of damage. Damage accumulation was quantified by the evolution of the Young's modulus throughout loading cycles. As mentioned before, viscoelastic nonlinearity was associated with hysteretic loops, asymmetric curves in tension/compression phases, and high TOFM magnitudes ( $\triangle \mathrm{TOFM}=5,40$, and $50 \mathrm{~ns}$ for bone samples A, B, and C, respectively). High levels of dissipative non-linearity were also observed, which confirmed the presence of microdamage. There is in fact no dissipative non-linearity in nondamaged materials.

The quadratic NL elastic parameter $\beta$ is obtained from a linear regression of the TOFM diagram 
(TOFM as function of instantaneous LF pressure). However, the calculation of $\beta$ also requires sample density and US pulse velocity measurements. These two parameters have a significant impact on $\beta$ amplitude (equation (4)). Since it is difficult to assess sample density, this parameter was fixed at $1180 \mathrm{~kg} / \mathrm{m}^{3}$. In contrast, the US pulse velocities were carefully measured, with values ranging from 1500 to $2200 \mathrm{~m} / \mathrm{s}$. However, due to the weight of the cubic velocity in the $\beta$ expression, the $|\beta|$ values measured were spread over a wide range of values (5-20 to more than 50). It was decided therefore to conclude that there was a strong NL behaviour (i.e. related to an actual microdamage) when the $|\beta|$ value was higher than 50. Results could have been presented as $\left(-L / L_{\text {sample }}\right) \times\left(\Delta \mathrm{TOFM} / \Delta p_{\mathrm{LF}}\right)$ only, but these would then be difficult to compare with data found in the literature. Thus, $|\beta|$ values less than 50 characterize classical NL behaviour (microdamage might be too weak to be detected with DAET). In contrast, the $|\beta|$ value higher than 50 cannot be explained only by a high material velocity value but is related to nonclassical non-linearity and is very probably due to microdamage. The NL behaviour was mostly associated with hysteresis, asymmetry, and significant dissipative non-linearity, characterizing non-classical non-linearity due to microcracks. These findings were always correlated with histology (sample 7, sample defatted by trichloroethylene, and sample Z).

All measurements were performed at least twice for each sample. DAET reproducibility was good for samples with low levels of non-linearity $(|\beta|<50$, $|\mathrm{TOFM}|<3 \mathrm{~ns})$. In samples exhibiting high levels of non-linearity $(|\beta|>50)$, DAET reproducibility was sample dependent. Some samples showed fairly constant behaviour from one test to the other, and others presented increased or decreased NL behaviour from one test to another. This can be partly explained by the well-known conditioning effect in damaged materials (viscoelastic memory effects). The NL response due to LF stress depends on the whole stress history of the sample. In future experiments particular care will be taken with DAET measurements, to take into account these possible conditioning effects.

A particular kind of damage was observed in the histology study, i.e. split trabeculae. To the authors' knowledge, no previous publication in the bone literature has reported such damage (or perhaps in Moore and Gibson [17]). Several aspects of slice preparation for confocal laser scanning microscopy analysis could be called into question. In fact, bone section size used in the present study was much thicker than those usually reported $(300 \mu \mathrm{m})$. However, in general, the confocal laser scanning microscopy results were in agreement with the DAET results. A surface density of microcracks greater than 0.5 per $\mathrm{mm}^{2}$ was associated with complex and high NL behaviour $(|\beta|$ values $>50)$.

\section{CONCLUSIONS}

Taken together, the results of the present study demonstrate that DAET is a sensitive method to detect microdamage in trabecular bone, although it is currently limited by the amount of bone crossed by the US pulses. However, these results could be improved by paying particular attention to the measurement chain, especially by optimizing a low-noise reception amplifier.

A fatigue protocol, combining DAET measurements before and after fatigue and sequential fluorochrome labelling, is currently being investigated. This will provide validation of DAET sensitivity to mechanically induced damage.

\section{ACKNOWLEDGEMENT}

The authors would like to thank Jean-Yves Tartu for his valuable contribution to the mechanical setup.

\section{REFERENCES}

1 Ammann, P. and Rizzoli, R. Bone strength and its determinants. Osteoporos. Int., 2003, 14, 13-18.

2 Colopy, S. A., Benz-Dean, J., Barrett, J. G., Sample, S. J., Lu, Y., Danova, N. A., Kalscheur, V. L., Vanderby, R., Markel, M. D., and Muir, P. Response of the osteocyte syncytium adjacent to and distant from linear microcracks during adaptation to cyclic fatigue loading. Bone, 2004, 35, 881-891.

3 Hazenberg, J. G., Taylor, D., and Lee, T. C. The role of osteocytes and bone microstructure in preventing osteoporotic fractures. Osteoporos. Int., 2007, 18, 1-8.

4 Burr, D. B., Turner, C. H., Naick, P., Forwood, M. R., Ambrosius, W., Hasan, M. S., and Pidaparti, R. Does microdamage accumulation affect the mechanical properties of bone? J. Biomech., 1998, 31, 337-345.

5 Zioupos, P. Accumulation of in-vivo fatigue microdamage and its relation to biomechanical properties in ageing human cortical bone. J. Microsc., 2001, 201, 270-278. 
6 Njeh, C. F., Boivin, C. M., and Langton, C. M. The role of ultrasound in the assessment of osteoporosis: a review. Osteoporos. Int., 1997, 7, 7-22.

7 Hans, D., Dargent-Molina, P., Schott, A. M., Sebert, J. L., Cormier, C., Kotzki, P. O., Delmas, P. D., Pouilles, J. M., Breart, G., and Meunier, P. J. Ultrasonographic heel measurements to predict hip fracture in elderly women: the EPIDOS prospective study. Lancet, 1996, 348, 511-514.

8 Renaud, G., Callé, S., Remenieras, J. P., and Defontaine, M. Exploration of trabecular bone nonlinear elasticity using time-of-flight modulation. IEEE Trans. Ultrason. Ferroelectrics Freq. Contr., 2008, 55, 1497-1507.

9 Sone, T., Imai, Y., Tomomitsu, T., and Fukunaga, M. Calcaneus as a site for the assessment of bone mass. Bone, 1998, 22, 155S-157S.

10 Vico, L., Lafage-Proust, M. H., and Alexandre, C. Effects of gravitational changes on the bone system in vitro and in vivo. Bone, 1998, 22, 95S-100S.

11 Mitton, D., Rappeneau, J., and Bardonnet, R. Effect of a supercritical $\mathrm{CO}_{2}$ based treatment on mechanical properties of human cancellous bone. Eur. J. Orthop. Surg. Traumatol., 2005, 15, 264-269.

12 Ichida, N., Sato, T., and Linzer, M. Imaging the nonlinear ultrasonic parameter of a medium. Ultrason. Imag., 1983, 5, 295-299.

13 Renaud, G., Callé, S., and Defontaine, M. Remote dynamic acoustoelastic testing: elastic and dissipative acoustic nonlinearities measured under hydrostatic tension and compression. Appl. Phys. Lett., 2009, 94, 011905.

14 Renaud, G., Callé, S., Remenieras, J. P., and Defontaine, M. Non-linear acoustic measurements to assess crack density in trabecular bone. In 11th
International Workshop on Nonlinear Elasticity in Materials. Int. J. Non-Linear Mech., 2008, 43, 194-200.

15 Nagaraja, S., Couse, T. L., and Guldberg, R. E. Trabecular bone microdamage and microstructural stresses under uniaxial compression. J. Biomech., 2005, 38, 707-716.

16 Rupprecht, M., Pogoda, P., Mumme, M., Rueger, J. M., Püschel, K., and Amling, M. Bone microarchitecture of the calcaneus and its changes in aging: a histomorphometric analysis of 60 human specimens. J. Orthop. Res., 2006, 24, 664-674.

17 Moore, T. L. and Gibson, L. J. Fatigue microdamage in bovine trabecular bone. J. Biomech. Engng, 2003, 125, 769-776.

18 Ostrovsky, L. A. and Johnson, P. A. Dynamic nonlinear elasticity in geomaterials. Nuovo Cimento Rivista Serie, 2001, 24, 70000-46.

19 Guyer, R. A. and Johnson, P. A. Nonlinear mesoscopic elasticity: evidence for a new class of materials. Phys. Today, 1999, 52, 30-35.

20 Van Den Abeele, K. and Visscher, J. D. Damage assessment in reinforced concrete using spectral and temporal nonlinear vibration techniques. $\mathrm{Ce}$ ment Concrete Res., 2000, 30, 1453-1464.

21 Muller, M., Sutin, A., Guyer, R. V., Talmant, M., Laugier, P., and Johnson, P. A. Nonlinear resonant ultrasound spectroscopy (NRUS) applied to damage assessment in bone. J. Acoust. Soc. Am., 2005, 118, 3946-3952.

22 Muller, M., Mitton, D., Moilanen, P., Bousson, V., Talmant, M., and Laugier, P. Prediction of bone mechanical properties using QUS and pQCT: study of the human distal radius. Med. Engng Phys., 2008, 30, 761-767. 